\title{
Simultaneous occurrence of multiple aetiologies of polycythaemia: renal cell carcinoma, sleep apnoea syndrome, and relative polycythaemia in a smoker with masked polycythaemia rubra vera
}

Lee-Yung Shih, Ming-Lun Wang, Jen-Fen Fu

\begin{abstract}
A 58 year old male heavy smoker presented with intracranial haemorrhage and erythrocytosis. Four aetiologies of polycythaemia-polycythaemia rubra vera (PRV), renal cell carcinoma, sleep apnoea syndrome, and relative polycythaemia-were found to be associated with the underlying causes of erythrocytosis. He did not fulfill the diagnostic criteria for PRV at initial presentation, but an erythropoietin independent erythroid progenitor assay identified the masked PRV, and the low postphlebotomy erythropoietin concentration also suggested the likelihood of PRV evolution. This case demonstrates that a search for all the possible causes of erythrocytosis is warranted in patients who already have one aetiology of polycythaemia.

(F Clin Pathol 2000;53:561-564)
\end{abstract}

Keywords: erythrocytosis; polycythaemia rubra vera; renal cell carcinoma; sleep apnoea syndrome; relative polycythaemia; endogenous erythroid colony

Polycythaemia includes a wide variety of different pathologies and can be divided into polycythaemia rubra vera (PRV), secondary polycythaemia, and relative polycythaemia. PRV is a myeloproliferative disease characterised by abnormal clonal proliferation of early haematopoietic stem cells. ${ }^{1}$ Secondary polycythaemia is associated with hypoxia or disorders with increased erythropoietin production. Patients with relative polycythaemia have normal red blood cell mass and decreased plasma volume. There is usually only one underlying mechanism of polycythaemia in an individual patient, although some patients do have two possible causes of polycythaemia (such as hypoxia/smoking and PRV); however, finding a third or even fourth possible cause is extremely unusual. We describe a patient who presented with cerebral haemorrhage and polycythaemia, which was found to be associated with four aetiologies: renal cell carcinoma, sleep apnoea syndrome, relative polycythaemia, and masked PRV. The features of our patient stress that all the potential causes of erythrocytosis should be continuously searched for, even when an apparent diagnosis has been made. In addition, we also demonstrated that in the presence of secondary causes, the possibility of masked PRV should be investigated by the in vitro erythroid progenitor study and/or post-phlebotomy serum erythropoietin concentrations to determine as precisely as possible all the underlying mechanisms of erythrocytosis, which allows greater confidence in the choice of therapeutic strategy, especially the use of myelosuppressive agents.

\section{Case report}

A 58 year old short obese man, with a snoring habit and daytime somnolence, had a history of hypertension and an episode of stroke one year ago. He had smoked one packet of cigarettes daily for more than 30 years. On 9 May 1996, the patient suddenly developed explosive headache and vomiting. $\mathrm{He}$ was initially managed at a community hospital and intracerebral haemorrhage was diagnosed after brain computed tomography (CT). The patient was then transferred to our hospital on 13 May. On examination, the body height was $166 \mathrm{~cm}$ and the body weight was $88 \mathrm{~kg}$. The blood pressure was $170 / 110 \mathrm{~mm} \mathrm{Hg}$. He was well oriented. The pupils were equal at $3 \mathrm{~mm}$ with brisk light reflex. The liver and spleen were not palpable in this obese man. The muscle strength was full in the right limbs and was $4+$ in the left limbs. The deep tendon reflexes were normal and symmetrical. The plantar response of the left side was equivocal and that of the right side was plantar flexion. The initial complete blood counts were: red blood cells, $8.58 \times 10^{12} /$ litre; haemoglobin, $225 \mathrm{~g} /$ litre; haematocrit, 0.699; mean corpuscular volume, $81.5 \mathrm{fl}$; platelets, $251 \times 10^{9} /$ litre; and white blood cells, $12.9 \times 10^{9} /$ litre with a normal differential count. Urinalysis revealed proteinuria (1500 mg/litre), and no evidence of haematuria. The arterial oxygen saturation was $97.6 \%, \mathrm{P}_{50}$ was $26.8 \mathrm{~mm} \mathrm{Hg}$, and carboxyhaemoglobin measured 10 days after cessation of smoking was $1.1 \%$. The total red cell volume (measured with ${ }^{51} \mathrm{Cr}$ ) after phlebotomy of $1000 \mathrm{ml}$ blood was 3.5 litres ( $42 \mathrm{ml} / \mathrm{kg}$ ), which was greater than $67 \%$ above the mean normal predicted value (reference value, 2.1 litres), ${ }^{2}$ and the calculated plasma volume was $2.1 \mathrm{li}$ tres (reference value, 3.0 litres). ${ }^{2}$ The leucocyte alkaline phosphatase score was 128 (reference value, 40-145). The vitamin B12 concentration was $582 \mathrm{pg} / \mathrm{ml}$ (reference value, 180-710). The bone marrow examination showed a hypercellular marrow with trilineage hyperplasia and absent marrow iron stores. A chest $x$ ray film revealed mild pulmonary congestion. The 
abdominal CT showed normal liver, no splenomegaly, and a mass measuring $4 \times 7 \mathrm{~cm}$ in the left kidney. The results of pulmonary function tests for spirometry, lung volume, and diffusing capacity were unremarkable. Overnight pulse oximetry revealed oxygen desaturation, with the lowest arterial oxygen saturation being only $79 \%$. The initial polysomnography showed a pattern of obstructive type sleep apnoea/hypopnoea, which occurred at a frequency of 46 episodes/sleep hour. The in vitro erythroid progenitor assay demonstrated the presence of erythropoietin independent erythroid colonies in both bone marrow and peripheral blood cultures. The serum erythropoietin concentration measured by radioimmunoassay was $4.48 \mathrm{U} /$ litre (normal range, 5.76-15.3). ${ }^{3} \mathrm{~A}$ diagnosis of masked PRV associated with renal tumour and obstructive sleep apnoea syndrome in conjunction with relative polycythaemia was made.

A total of $1600 \mathrm{ml}$ blood was removed over two weeks. On 27 May, the blood counts were: haemoglobin, $182 \mathrm{~g} /$ litre; haematocrit, 0.549; red blood cells, $6.50 \times 10^{12} /$ litre; platelets, $225 \times 10^{9} /$ litre; and white blood cells, $8.0 \times 10^{9} \%$ litre. The patient underwent left nephrectomy with an estimated blood loss of $1500 \mathrm{ml}$ during surgery on 31 May 1996. The pathological diagnosis was renal cell carcinoma. The patient stopped smoking after admission and his hypertension was treated successfully with atenolol. The haematocrit value declined to 0.364 postoperatively, gradually returned to normal, and then rose to 0.56 in September 1996, when white blood cell and platelet counts were still within normal limits. $\mathrm{He}$ required periodic phlebotomy subsequently to keep haematocrit values below 0.45. In May 1997, the blood counts showed haemoglobin, $145 \mathrm{~g} /$ litre; haematocrit, 0.466 ; white blood cells, $12.7 \times 10^{9} /$ litre; platelets, $569 \times 10^{9} /$ litre; and serum erythropoietin, <2 U/litre. Abdominal CT on May 27 showed mild splenomegaly and no evidence of recurrence of the renal tumour. His weight had fallen by $6 \mathrm{~kg}$. Follow up polysomnography revealed that the frequency of apnoea/hypopnoea was reduced from 46 episodes/sleep hour to 15 episodes/ sleep hour. A follow up blood volume measurement revealed a restoration of plasma volume
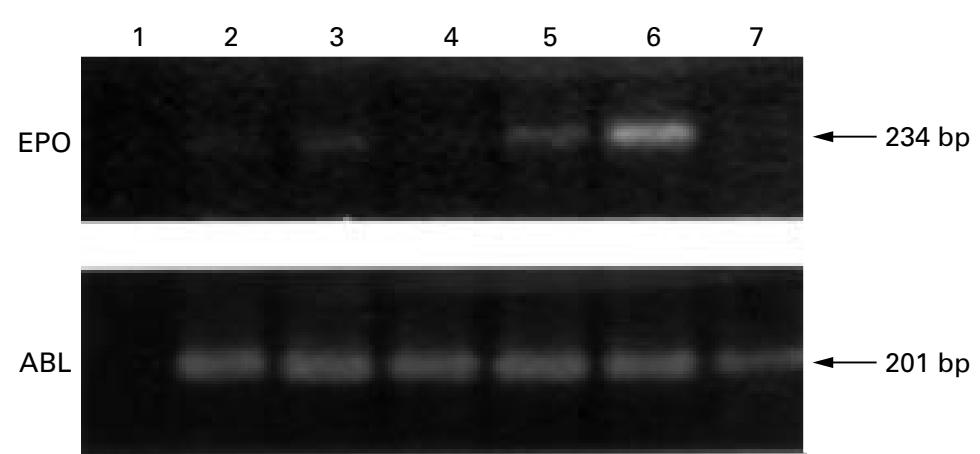

Figure 1 Reverse transcription polymerase chain reaction for erythropoietin $m R N A$ from our patient (lane 2), two patients with renal cell carcinoma but without erythrocytosis (lanes 3 and 4), normal renal tissue (lanes 5 and 6), negative controls (no RNA in lane 1 and HL-60 cell line in lane 7). ABL indicates amplification of $c A B L$ in all lanes except lane 1. to normal (3.3 litres). Myelosuppressive treatment with hydroxyurea was initiated later because of a history of cerebrovascular complications in conjunction with persistent pancytosis and increasing phlebotomy requirement during follow up.

ERYTHROID PROGENITOR ASSAY

Serum containing semisolid cultures for BFU-E (burst forming units-erythroid) were performed as described previously. ${ }^{4}$ Serum free cultures were grown using a modification of the technique of Sonoda et al. ${ }^{5}$ Briefly, nonadherent $\mathrm{T}$ cell depleted cells were plated in $1.2 \%$ methylcellulose Iscove's modified Dulbecco's medium (IMDM) containing a lipid mixture (lecithin, cholesterol, and linoleic acid), $450 \mu \mathrm{g} / \mathrm{ml}$ transferrin saturated with $\mathrm{FeCl}_{3}, 5 \times 10^{-5} \mathrm{M} 2$ mercaptoethanol, $1 \%$ deionised fatty acid free and globulin free bovine serum albumin without the addition of erythropoietin or other haematopoietic growth factors. The dishes were incubated at $37^{\circ} \mathrm{C}$ in a humidified atmosphere of $5 \% \mathrm{CO}_{2}$ in air. Erythroid colonies were scored for BFU-E by their red colour on an inverted microscope on day 14 .

The number of erythropoietin independent BFU-E (endogenous erythroid colonies; EEC) in bone marrow was $251 / 5 \times 10^{4}$ non-adherent $\mathrm{T}$ cell depleted cells, and in peripheral blood the numbers were 22 and 10 in serum containing and serum free culture, respectively. Normal subjects or patients with secondary polycythaemia never form EEC in our culture system. ${ }^{3}$

DETECTION OF MRNA FOR ERYTHROPOIETIN IN RENAL TUMOUR BY REVERSE TRANSCRIPTION POLYMERASE CHAIN REACTION (RT-PCR)

Frozen tissue samples were homogenised and RNA was extracted with Trizol reagent (Life Technologies, Gibco, Gaithersburg, Maryland, USA). To check the integrity of the RNA, aliquots were run on a $1.1 \%$ formaldehyde/agarose gel. After initial denaturation, $2 \mu \mathrm{g}$ RNA was combined with $0.15 \mu \mathrm{g}$ random hexamers, RT buffer, $0.01 \mathrm{M}$ dithiothreitol, $250 \mu \mathrm{M}$ dNTP, 20 U placental RNase inhibitor (HT Biotechnology, Cambridge, UK), 400 U Moloney murine leukaemia virus reverse transcriptase (Life Technologies, Gibco). RT was performed at $37^{\circ} \mathrm{C}$ for two hours. The cDNA products $(25 \%)$ were amplified with $0.025 \mathrm{U} / \mu \mathrm{l}$ Taq polymerase (Perkin Elmer) in PCR buffer, $0.2 \mathrm{mM}$ dNTP, $2 \mu \mathrm{M}$ of $5^{\prime}$ primer (5'-CCC TGT TGG TCA ACT CTT CC-3') and 3' primer (5'-GTG TAC AGC TTC AGC TTT CC- $\left.3^{\prime}\right) .^{6}$ The PCR reaction was performed in a 9600 thermal cycler (Perkin Elmer Cetus, Norwalk, Connecticut, USA) with a step programme consisting of initial denaturation at $94^{\circ} \mathrm{C}$ for one minute, annealing at $56^{\circ} \mathrm{C}$ for one minute, and extension at $72^{\circ} \mathrm{C}$ for two minutes for a total of 30 cycles, and extension for 10 minutes at $72^{\circ} \mathrm{C}$ after the last cycle. PCR products were run on a $2 \%$ NuSieve agarose gel and stained with ethidium bromide. Samples from two patients with renal cell carcinoma without erythrocytosis and another two 
samples of normal renal tissues were used as controls. Every sample was tested for RT and PCR efficiency by using primers for the ABL gene (5' primer: 5'-TTC AGC GGC CAG TAG CAT CTG ACT T-3'; 3' primer: 5'-AGA TAC TCA GCG GCA TTG-3').

We failed to demonstrate increased expression of mRNA for erythropoietin in the patient's renal tumour compared with control tissues (fig 1).

\section{Discussion}

Vascular complications are the leading cause of death in patients with PRV. Those who received adequate treatment with phlebotomy and/or cytotoxic agents had a median survival of $10-13$ years compared with only 18 months in untreated patients. In contrast, patients with benign pure erythrocytosis might not require treatment because no increased risk of vascular accidents was seen compared with the normal population. ${ }^{7}$ On the other hand, there are many physiological and pathological states, often treatable, associated with secondary polycythaemia. Thus, it is clinically important to define precisely the causes of polycythaemia at presentation for each patient and treat them appropriately, which will favourably affect the outcome.

At initial evaluation, our patient had absolute erythrocytosis with two disorders known to be associated with secondary erythrocytosis. $\mathrm{He}$ had no splenomegaly or thrombocytosis, the white blood cell count returned to a normal value two weeks after cerebral haemorrhage, and the vitamin B12 value and leucocyte alkaline phosphatase score were within normal limits. He did not fulfill the diagnostic criteria for PRV at presentation. Although the diagnostic criteria of PRV proposed by the polycythaemia vera study group are useful and relatively easy to apply, with a false positive rate of less than $0.5,{ }^{7}$ there are some instances in which they might not be sufficient. The patient's bone marrow and blood cells grew erythroid colonies in vitro in the absence of exogenous erythropoietin, even in a serum free culture system. EECs are never present in normal subjects or patients with secondary polycythaemia in our culture system. ${ }^{3}$ We also demonstrated that the EEC assay can identify at presentation those patients with idiopathic erythrocytosis who will later progress to PRV and those who will remain in benign pure erythrocytosis. ${ }^{8}$ Positivity for EEC growth in this patient allowed the earlier identification of masked PRV as an additional aetiology of polycythaemia, which was not clinically apparent until one year after presentation when he had pancytosis, splenomegaly, and required periodic phlebotomy followed by myelosuppressive treatment.

Our recent study showed that the postphlebotomy serum erythropoietin concentration at a cut off value of $<9$ U/litre can distinguish PRV from unclassifiable polycythaemia. ${ }^{3}$ After removal of the renal tumour and alleviation of sleep apnoea syndrome, the postphlebotomy serum erythropoietin concentration in our patient was still very low; this observation further validated our previous findings. Because the clonogenic cultures are technically demanding and are not available in most routine laboratories, measurement of the post-phlebotomy serum erythropoietin concentration, which is a simple and readily accessible test, could be an alternative when the endogenous erythroid assay is not available. ${ }^{3}$

Abdominal ultrasonography or CT, which can detect the presence of relevant liver and renal pathology, is essential in all patients with polycythaemia. In addition, these imaging studies can accurately determine splenic size in obese patients such as ours. Several renal diseases may be complicated by erythrocytosis. Careful examination of the kidneys is mandatory to detect renal lesions in patients with polycythaemia. A large renal tumour was disclosed by our proposed stepwise approach. The incidence of erythrocytosis in patients with renal cell carcinoma is $1-5 \%$, and approximately $4 \%$ of patients with erythrocytosis have renal cell carcinoma, ${ }^{9}$ especially those who have large tumours. Our patient had a low serum erythropoietin value. Patients with renal cell carcinoma and erythrocytosis do not necessarily have a raised serum erythropoietin concentration. Renal vein sampling for erythropoietin, which would be more specific than the serum value, was not performed in our patient. Successful surgical removal of renal tumours results in resolution of polycythaemia in patients without other causes of erythrocytosis. ${ }^{10}$ In contrast, removal of the incidentally found renal cell carcinoma had no apparent effect on erythrocytosis in our patient. Using RT-PCR analysis, we found that the patient's renal tumour did not have increased erythropoietin mRNA expression when compared with normal renal tissues and renal tumours without erythrocytosis. This observation suggested that his renal cell carcinoma did not contribute greatly to the erythrocytosis.

Approximately $5-10 \%$ of patients with nocturnal apnoea have polycythaemia. Nocturnal oxygen desaturation with normal daytime values has been shown to be the cause of polycythaemia in $10-20 \%$ of patients with unexplained erythrocytosis. ${ }^{11}$ It is important to take a careful history, especially in obese patients, about symptoms of nocturnal oxygen desaturation, including snoring, nocturnal restlessness, and day time somnolence. In addition to the presence of these clinical manifestations, overnight pulse oximetry followed by polysomnography confirmed the sleep apnoea syndrome in our patient. Weight reduction resulted in a decrease in the frequency of apnoeic/ hypopnoeic episodes during sleep in our patient. All overweight patients with the sleep apnoea phenomenon should be encouraged to lose weight. It was not clear to what extent the sleep apnoea contributed to erythrocytosis in our patient, who already had other causes of polycythaemia at initial evaluation. Normal pulmonary function tests excluded the possibility of chronic obstructive lung disease as a further cause of erythrocytosis.

Our patient had been a heavy smoker, but abstained from smoking after hospitalisation 
when the carboxyhaemoglobin value was not raised. Smoking can produce absolute polycythaemia, relative polycythaemia, or a combination thereof. ${ }^{12}$ Smith and Landaw found that most smokers with polycythaemia have a reduced plasma volume. ${ }^{12}$ Our patient had a plasma volume $30 \%$ below the normal predicted value, which indicated that relative polycythaemia also played a role in his underlying polycythaemia. Smoking and sleep apnoea syndrome as well as hypertension could induce plasma volume contraction, ${ }^{13}$ although we were not certain whether these disorders were (or to what extent) linked to his erythrocytosis at initial presentation. Cessation of smoking, successful antihypertensive treatment, and weight reduction, along with a decrease in the frequency of sleep apnoea episodes, restored his plasma volume to normal.

This case demonstrates that more than one aetiology may concurrently contribute to the development of erythrocytosis in a single patient. However, it was difficult to delineate the proportional effects of the four causes on the erythrocytosis at initial presentation in our patient. The proposed comprehensive stepwise investigations, based on the knowledge of both physiology and pathology about all causes of erythrocytosis, are crucial for making an accurate diagnosis and choosing the appropriate management in patients with polycythaemia. This structured approach allows completeness of diagnosis, and in this case, resection of a renal tumour, which might have caused serious problems if left undiagnosed. In the absence of satisfactory criteria for PRV, the assessment of endogenous erythroid colonies and/or the post-phlebotomy serum erythropoietin concentration may serve as useful tools to uncover masked PRV before its progression to full blown disease. At the present time, the erythroid progenitor assay is not readily available to most physicians. The postphlebotomy serum erythropoietin value, which gives a high sensitivity, specificity, positive predictive value, and negative predictive value in the differentiation of patients with pure erythrocytosis who will progress to PRV from who will not, is especially helpful in clinical practice. ${ }^{3}$ Some patients with idiopathic erythrocytosis, such as our patient, might evolve to PRV and have a higher risk of developing major vascular complications, while others run a benign course. The early identification of all the possible causes of erythrocytosis is very important to guide therapeutic decisions, which will considerably reduce the morbidity and mortality associated with polycythaemia.

This work was partly supported by grant number CMRP859 from Chang Gung Memorial Hospital and by NSC88-2314B182-014 from the National Science Council, Taiwan. The authors thank Dr CK Chuang for providing the frozen renal tumour sample from this patient for the erythropoietin mRNA assay.

1 Adamson JW, Fialkow PJ, Murphy S, et al. Polycythemia vera: stem-cell and probable clonal origin of the disease. $N$ Engl f Med 1976;295:913-16.

2 Pearson TC, Guthrie DL, Simpson J, et al. Interpretation of measured red cell mass and plasma volume in adults. $\mathrm{Br} \mathcal{F}$ Haematol 1995;89:748-56.

3 Shih LY, Lee CT, See LC, et al. In vitro culture growth of erythroid progenitors and serum erythropoietin assay in the differential diagnosis of polycythemia. Eur 7 Clin Invest 1998;28:569-76.

4 Shih LY, Lee CT. Identification of masked polycythemia vera from patients with idiopathic marked thrombocytosis by endogenous erythroid colony assay. Blood 1994;83:744-

5 Sonoda Y, Yang YC, Wong GG, et al. Analysis in serum-free culture of the targets of recombinant human hemopoietic growth factors: interleukin 3 and granulocyte/macrophagecolony-stimulating factor are specific for early developmental stages. Proc Natl Acad Sci U S A 1988;85:4360-4.

6 Juul SE, Yachnis AT, Christensen RD. Tissue distribution of erythropoietin and erythropoietin receptor in the developing human fetus. Early Hum Dev 1998;52:235-49.

7 Berk PD, Goldberg JD, Donovan PB, et al. Therapeutic recommendations in polycythemia vera based on polyommendations in polycythemia vera based on poly23:132-43.

8 Shih LY, Lee CT, Ou YC. Prediction of clinical course in patients with idiopathic erythrocytosis by endogenous erythroid colony assay but not by serum erythropoietin levels. Exp Hematol 1997;25:288-92.

9 Orwoll ES, Orwoll RL. Hematologic abnormalities associated with renal cell carcinoma. Hematol Oncol Clin North Am 1987;1:247-8.

10 Moore-Gillon JC, Treacher DF, Gaminara EJ, et al. Intermittent hypoxia in patients with unexplained polycythemia. BMF 1986;293:588-90.

11 Damon A, Holub DA, Melicow MM. Polycythemia and renal carcinoma: report of ten new cases, two with long hematologic remission following nephrectomy. Am f Med 1958;25:182-97.

12 Smith JR, Landaw SA. Smokers' polycythemia. N Engl f Med 1978;298:6-10.

13 Weinreb NJ. Relative polycythemia. In: Wasserman LR, Berk PD, Berlin NI, eds. Polycythemia vera and the myeloproliferative disorders. Philadelphia: WB Saunders, 1995:226-58 